\title{
On the dynamics of a five-order fuzzy difference equation
}

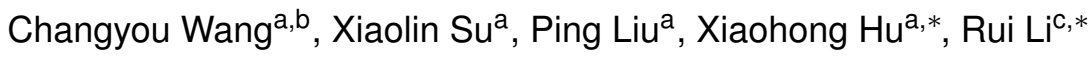 \\ ${ }^{a}$ College of Science, Chongqing University of Posts and Telecommunications, Chongqing 400065, P. R. China. \\ ${ }^{b}$ School of Applied Mathematics, Chengdu University of Information Technology, Chengdu, Sichuan 610225, P. R. China. \\ ${ }^{c}$ College of Automation, Chongqing University of Posts and Telecommunications, Chongqing 400065, P. R. China.
}

Communicated by K. Q. Lan

\begin{abstract}
Our aim in this paper is to investigate the existence and uniqueness of the positive solutions and the asymptotic behavior of the equilibrium points of the fuzzy difference equation

$$
x_{n+1}=\frac{A x_{n-1} x_{n-2}}{D+B x_{n-3}+C x_{n-4}}, \quad n=0,1,2, \cdots,
$$

where $x_{n}$ is a sequence of positive fuzzy numbers, the parameters $A, B, C, D$ and the initial conditions $x_{-4}, x_{-3}, x_{-2}, x_{-1}, x_{0}$ are positive fuzzy numbers. Moreover, some numerical examples to the difference system are given to verify our theoretical results. (C)2017 All rights reserved.
\end{abstract}

Keywords: Fuzzy difference equation, existence, uniqueness, equilibrium point, asymptotic behavior. 2010 MSC: 39A11, 03E72.

\section{Introduction}

Because of the necessity for some techniques that can be used in mathematical models describing the real world phenomenon, nonlinear difference equation have been studied in the fields of population biology, economics, probability theory, genetics, psychology etc., (see, e.g., [4, 10, 21, 22, 25] and the references therein). In recent years, with the dramatically development of computer-based computational techniques, difference equation is found to be much appropriate mathematical representation for computer simulation and experiment (see, e.g., [9, 26-29] and the references therein). However, in view of the facts that the information of the difference equation model to describe many practical problems is incomplete and the fuzzy set theory is a powerful tool for modeling uncertainty and for processing vague or subjective information in mathematical model, it is more interesting to investigate the behavior of solutions of a system of fuzzy difference equation where the parameters and the initial values are fuzzy numbers and its solutions are sequences of fuzzy numbers (see, e.g., $[1,7,11,12,15-18]$ and the references therein).

\footnotetext{
*Corresponding author

Email addresses: huxh@cqupt.edu.cn (Xiaohong Hu), liruimath@qq.com (Rui Li)
} 
Making a historical flash back for the equation we study in this paper, we should mention that in 1996, Deeba et al. [6] studied the first order difference equation

$$
x_{n+1}=w x_{n}+q, \quad n=0,1, \cdots,
$$

where $x_{n}$ is a sequence of fuzzy numbers and $x_{0}, q, w$ are fuzzy numbers, which arise in population genetics. Moreover, Deeba and Korvin [5] studied the following second order linear fuzzy difference equation

$$
C_{n+1}=C_{n}-a b C_{n-1}+m, \quad n=0,1, \cdots,
$$

where $C_{n}$ is a sequence of fuzzy numbers and $a, b, m, C_{0}, C_{1}$ are fuzzy numbers. This equation is a linearized model of a nonlinear model which determines the carbon dioxide (CO2) level in the blood.

In 2003, Papaschinopoulos and Stefanidou [20] studied the existence, the uniqueness, the boundedness and persistence of the positive solutions of the following fuzzy difference equation

$$
x_{n+1}=\sum_{i=0}^{k} \frac{A_{i}}{x_{n-i}^{p_{i}}}, \quad n=0,1, \cdots,
$$

where $k \in\{1,2, \cdots\}$, the parameters $A_{i}, i \in\{0,1, \cdots, k\}$ are positive fuzzy numbers, the parameters $p_{i}, i \in\{0,1, \cdots, k\}$ are positive real constants and the initial values $x_{i}, i \in\{-k,-k+1, \cdots, 0\}$ are positive fuzzy numbers. Moreover, in 2006, they [24] considered the periodicity of the positive solutions of the following max-type fuzzy difference equation

$$
x_{n+1}=\max \left\{\frac{A_{0}}{x_{n-k}}, \frac{A_{1}}{x_{n-m}}\right\}, \quad n=0,1, \cdots,
$$

where $k, m$ are positive integers, $A_{0}, A_{1}$ and the initial values $x_{i}, i \in\{-d,-d+1, \cdots,-1\}, d=\max \{k, m\}$ are positive fuzzy numbers.

Recently, Zhang et al. [33] studied the existence, asymptotic behavior of the positive solutions of a fuzzy nonlinear difference equation

$$
x_{n+1}=\frac{A x_{n}+x_{n-1}}{B+x_{n-1}}, n=0,1,2, \cdots,
$$

where $\left\{x_{n}\right\}$ is a sequence of positive fuzzy number, $A, B$ are positive fuzzy numbers and the initial conditions $x_{-1}, x_{0}$ are positive fuzzy numbers. Moreover, in 2014, Zhang et al [34] continuously dealt with the existence, the boundedness and the asymptotic behavior of the positive solutions for a first order fuzzy Ricatti difference equation

$$
x_{n+1}=\frac{A+x_{n}}{B+x_{n}}, n=0,1,2, \cdots,
$$

where $\left\{x_{n}\right\}$ is a sequence of positive fuzzy numbers, $A, B$ and the initial value $x_{0}$ are positive fuzzy numbers.

More recently, in 2015, Zhang et al. [32] investigated the boundedness, persistence and global behavior of a positive fuzzy solution of the third-order rational fuzzy difference equation

$$
x_{n+1}=A+\frac{x_{n-1}}{x_{n-1} x_{n-2}}, \quad n=0,1,2, \cdots,
$$

where $A$ and initial values $x_{0}, x_{-1}, x_{-2}$ are positive fuzzy numbers. In 2017, Khastan [13] considered the existence, uniqueness and global behavior of the solution for the following two inequivalent fuzzy difference equations

$$
x_{n+1}-q=w x_{n}, \quad n=0,1, \cdots .
$$


Motivated by the discussions above, this paper aims at studying the existence and uniqueness of the positive solutions and the asymptotic behavior of the equilibrium points of the following five-order fuzzy nonlinear difference equation

$$
x_{n+1}=\frac{A x_{n-1} x_{n-2}}{D+B x_{n-3}+C x_{n-4}}, \quad n=0,1,2, \cdots,
$$

where $\left\{x_{n}\right\}$ is a sequence of positive fuzzy numbers, $A, B, C, D$ and the initial conditions $x_{-4}, x_{-3}, x_{-2}$, $x_{-1}, x_{0}$ are positive fuzzy numbers. When the parameters and the initial values are positive real numbers, Wang et al. [30] considered the global attractivity of the equilibrium point, and the asymptotic behavior of the solutions of the difference equation (1.1).

This paper is arranged as follows: in Section 2, we give some definitions and preliminary results. The main results and their proofs are given in Section 3. Finally, some numerical simulations are given to illustrate our theoretical analysis.

\section{Preliminaries and notations}

For the convenience of the readers, we give the following definitions and preliminary results, see $[2,3,8,14,23]$.

Definition 2.1. For a set $B$ we denote by $\bar{B}$ the closure of $B$. We say that a function $A: R \rightarrow[0,1]$ is a fuzzy number if it satisfies the following properties

(i) $A$ is normal, i.e., there exists $x \in R$ such that $A(x)=1$;

(ii) $A$ is a fuzzy convex, i.e., $A\left(t x_{1}+(1-t) x_{2}\right) \geqslant \min \left\{A\left(x_{1}\right), A\left(x_{2}\right)\right\}$, for all $t \in[0,1], x_{1}, x_{2} \in R$;

(iii) $A$ is upper semicontinuous on $R$;

(iv) $A$ is compactly supported, i.e., supp $A=\overline{\cup_{\alpha \in(0,1]}[A]_{\alpha}}=\overline{\{x \in R: A(x)>0\}}$ is compact.

Let us denote by $R_{f}$ the set of all fuzzy numbers. For $\alpha \in(0,1]$ and $A \in R_{f}$, we denote $\alpha$-cuts of fuzzy number $A$ by $[A]_{\alpha}=\{x \in R: A(x) \geqslant \alpha\}$ and $[A]_{0}=\overline{\{x \in R: A(x)>0\}}$. We call $[A]_{0}$ the support of fuzzy number $A$ and denote it by supp $(u)$. It is clear that the $[A]_{\alpha}$ is a bounded closed interval in $R$, we say that a fuzzy number $A$ is positive if supp $A \subset(0, \infty)$. It is obvious that if $A$ is a positive real number (trivial fuzzy number), then $A$ is a positive fuzzy number with $[A]_{\alpha}=[A, A]$. For $u, v \in R_{f},[u]_{\alpha}=\left[u_{l, \alpha}, u_{r, \alpha}\right]$, $[v]_{\alpha}=\left[v_{l, \alpha}, v_{r, \alpha}\right]$, and $\lambda \in R$, the sum $\mu+v$, the scalar product $\lambda \mu$, multiplication $u v$ and division $\frac{u}{v}$ in the standard interval arithmetic (SIA) setting are defined by

$$
\begin{gathered}
{[\mu+v]_{\alpha}=[\mu]_{\alpha}+[v]_{\alpha}, \quad[\lambda \mu]_{\alpha}=\lambda[\mu]_{\alpha}, \quad \forall \alpha \in[0,1],} \\
{[u v]_{\alpha}=\left[\min \left\{u_{l, \alpha} v_{l, \alpha}, u_{l, \alpha} v_{r, \alpha}, u_{r, \alpha} v_{l, \alpha}, u_{r, \alpha} v_{r, \alpha}\right\}, \max \left\{u_{l, \alpha} v_{l, \alpha}, u_{l, \alpha} v_{r, \alpha}, u_{r, \alpha} v_{l, \alpha}, u_{r, \alpha} v_{r, \alpha}\right\},\right.} \\
{\left[\frac{u}{v}\right]_{\alpha}=\left[\min \left\{\frac{u_{l, \alpha}}{v_{l, \alpha}}, \frac{u_{l, \alpha}}{v_{r, \alpha}}, \frac{u_{r, \alpha}}{v_{l, \alpha}}, \frac{u_{r, \alpha}}{v_{r, \alpha}}\right\}, \max \left\{\frac{u_{l, \alpha}}{v_{l, \alpha}}, \frac{u_{l, \alpha}}{v_{r, \alpha}}, \frac{u_{r, \alpha}}{v_{l, \alpha}}, \frac{u_{r, \alpha}}{v_{r, \alpha}}\right\}\right], \quad 0 \notin[v]_{\alpha} .}
\end{gathered}
$$

Definition 2.2. Let $u, v$ be fuzzy numbers with $[u]_{\alpha}=\left[u_{l, \alpha}, u_{r, \alpha}\right],[v]_{\alpha}=\left[v_{l, \alpha}, v_{r, \alpha}\right], \alpha \in[0,1]$. Then we define the metric on the fuzzy numbers set as follows

$$
\mathrm{D}(\mathrm{u}, v)=\sup \max \left\{\left|\mathrm{u}_{\mathrm{l}, \alpha}-v_{\mathrm{l}, \alpha}\right|,\left|\mathrm{u}_{\mathrm{r}, \alpha}-v_{\mathrm{r}, \alpha}\right|\right\},
$$

where sup is taken for all $\alpha \in[0,1]$. Then $\left(R_{f}, D\right)$ is a complete metric space. For future use we define $\hat{0} \in R_{f}$ as

$$
\hat{0}(x)= \begin{cases}1, & x=0 \\ 0, & x \neq 0\end{cases}
$$

Thus, $[\hat{0}]_{\alpha}=[0,0], 0<\alpha \leqslant 1$. 
Lemma 2.3. Let $\mathrm{I}_{x}, \mathrm{I}_{y}$ be some intervals of real numbers and let $\mathrm{f}: \mathrm{I}_{x}^{\mathrm{k}+1} \times \mathrm{I}_{y}^{\mathrm{l}+1} \rightarrow \mathrm{I}_{x}, \mathrm{~g}: \mathrm{I}_{x}^{\mathrm{k}+1} \times \mathrm{I}_{y}^{\mathrm{l}+1} \rightarrow \mathrm{I}_{y}$ be continuously differentiable functions. Then for every set of initial conditions $\left(x_{i}, y_{j}\right) \in I_{x} \times I_{y},(i=-k,-k+$ $1, \cdots, 0, j=-l,-l+1, \cdots, 0)$, the following system of difference equations

$$
\left\{\begin{array}{l}
x_{n+1}=f\left(x_{n}, x_{n-1}, \cdots, x_{n-k}, y_{n}, y_{n-1}, \cdots, y_{n-l}\right), \\
y_{n+1}=g\left(x_{n}, x_{n-1}, \cdots, x_{n-k}, y_{n}, y_{n-1}, \cdots, y_{n-l}\right),
\end{array} \quad n=0,1,2, \cdots,\right.
$$

has a unique solution $\left\{\left(x_{i}, y_{j}\right)\right\}_{i=-k, j=-l}^{+\infty,+\infty}$.

Definition 2.4. A point $(\bar{x}, \bar{y}) \in I_{x} \times I_{y}$ is called an equilibrium point of system (2.1) if

$$
\bar{x}=f(\bar{x}, \bar{x}, \cdots, \bar{x}, \bar{y}, \bar{y}, \cdots, \bar{y}), \quad \bar{y}=g(\bar{x}, \bar{x}, \cdots, \bar{x}, \bar{y}, \bar{y}, \cdots, \bar{y}) .
$$

That is, $\left(x_{n}, y_{n}\right)=(\bar{x}, \bar{y})$ for $n \geqslant 0$ is the solution of difference system $(2.1)$, or equivalently, $(\bar{x}, \bar{y})$ is a fixed point of the vector map $(f, g)$.

Definition 2.5. Assume that $(\bar{x}, \bar{y})$ is an equilibrium point of the system (2.1). Then, we have

(i) An equilibrium point $(\bar{x}, \bar{y})$ is called locally stable, if for every $\varepsilon>0$, there exists $\delta>0$ such that for any initial conditions $\left(x_{i}, y_{i}\right) \in I_{x} \times I_{y},(i=-k, \cdots, 0, j=-l, \cdots, 0)$, with $\sum_{i=-k}^{0}\left|x_{i}-\bar{x}\right|<$ $\delta, \sum_{j=-l}^{0}\left|y_{j}-\bar{y}\right|<\delta$, we have $\left|x_{n}-\bar{x}\right|<\varepsilon,\left|y_{n}-\bar{y}\right|<\varepsilon$ for any $n>0$.

(ii) An equilibrium point $(\bar{x}, \bar{y})$ is called attractor, if $\lim _{n \rightarrow \infty} x_{n}=\bar{x}, \lim _{n \rightarrow \infty} y_{n}=\bar{y}$ for any initial conditions $\left(x_{i}, y_{i}\right) \in I_{x} \times I_{y},(i=-k, \cdots, 0, j=-l, \cdots, 0)$.

(iii) An equilibrium point $(\bar{x}, \bar{y})$ is called asymptotically stable, if it is stable, and is also attractor.

(iv) An equilibrium point $(\bar{x}, \bar{y})$ is called unstable, if it is not locally stable.

Definition 2.6. Let $(\bar{x}, \bar{y})$ be an equilibrium point of the vector map $F=\left(f, x_{n}, \cdots, x_{n-k}, g, y_{n}, \cdots, y_{n-l}\right)$, where $f$ and $g$ are continuously differentiable functions at $(\bar{x}, \bar{y})$. The linearized system of $(2.1)$ about the equilibrium point $(\bar{x}, \bar{y})$ is $X_{n+1}=F\left(X_{n}\right)=F_{j} \cdot X_{n}$, where $F_{J}$ is the Jacobian matrix of the system (2.1) about $(\bar{x}, \bar{y})$ and $x_{n}=\left(x_{n}, \cdots, x_{n-k}, y_{n}, \cdots, y_{n-l}\right)^{\top}$.

Definition 2.7. let $p, q, s, t$ be four nonnegative integers such that $p+q=n, s+t=m$. Split $x=$ $\left(x_{1}, x_{2}, \cdots, x_{n}\right)$ into $x=\left([x]_{p},[x]_{q}\right)$ and $y=\left(y_{1}, y_{2}, \cdots, y_{m}\right)$ into $y=\left([y]_{s},[y]_{t}\right)$, where $[x]_{\sigma}$ denotes a vector with $\sigma$-components of $x$. We say that the function $f\left(x_{1}, x_{2}, \cdots, x_{n}, y_{1}, y_{2}, \cdots, y_{m}\right)$ possesses a mixed monotone property in subsets $I_{x}^{n} \times I_{y}^{m}$ of $R^{n} \times R^{m}$, if $f\left([x]_{p},[x]_{q},[y]_{s},[y]_{t}\right)$ is monotone non-decreasing in each component of $\left([x]_{p},[y]_{s}\right)$, and is monotone non-increasing in each component of $\left([x]_{q},[y]_{t}\right)$ for $(x, y) \in I_{x}^{n} \times I_{y}^{m}$. In particular, if $q=0, t=0$, then it is said to be monotone non-decreasing in $I_{x}^{n} \times I_{y}^{m}$.

Lemma 2.8. Assume that $\mathrm{X}(\mathrm{n}+1)=\mathrm{F}(\mathrm{X}(\mathrm{n})), \mathrm{n}=0,1, \cdots$ is a system of difference equations and $\overline{\mathrm{X}}$ is the equilibrium point of this system i.e., $\mathrm{F}(\overline{\mathrm{X}})=\overline{\mathrm{X}}$. Then we have

(i) If all eigenvalues of the Jacobian matrix $\mathrm{J}_{\mathrm{F}}$ about $\overline{\mathrm{X}}$ lie inside the open unit disk $|\lambda|<1$, then $\overline{\mathrm{X}}$ is locally asymptotically stable.

(ii) If one of eigenvalues of the Jacobian matrix $\mathrm{J}_{\mathrm{F}}$ about $\overline{\mathrm{X}}$ has norm greater than one, then $\overline{\mathrm{X}}$ is unstable.

Lemma 2.9. Assume that $X(n+1)=F(X(n)), n=0,1, \cdots$, is a system of difference equations and $\bar{X}$ is the equilibrium point of this system, the characteristic polynomial of this system about the equilibrium point $\bar{X}$ is 
$P(\lambda)=a_{0} \lambda^{n}+a_{1} \lambda^{n-1}+\cdots+a_{n-1} \lambda+a_{n}=0$, with the real coefficients and $a_{0}>0$. Then all roots of the polynomial $\mathrm{P}(\lambda)$ lie inside the open unit disk $|\lambda|<1$ if and only if

$$
\Delta_{\mathrm{k}}>0, \text { for } \mathrm{k}=1,2, \cdots, \mathrm{n},
$$

where $\Delta_{\mathrm{k}}$ is the principal minor of order $\mathrm{k}$ of the $\mathrm{n} \times \mathrm{n}$ matrix

$$
\Delta_{n}=\left[\begin{array}{ccccc}
a_{1} & a_{3} & a_{5} & \cdots & 0 \\
a_{0} & a_{2} & a_{4} & \cdots & 0 \\
0 & a_{1} & a_{3} & \cdots & 0 \\
\vdots & \vdots & \vdots & \ddots & \vdots \\
0 & 0 & 0 & \cdots & a_{n}
\end{array}\right]
$$

\section{Main results}

First we study the existence and uniqueness of the positive solutions of (1.1), we need the following lemmas.

Lemma 3.1 ([19]). Let $\mathrm{f}$ be a continuous function from $\mathrm{R}^{+} \times \mathrm{R}^{+} \times \mathrm{R}^{+}$into $\mathrm{R}^{+}$and $\mathrm{A}, \mathrm{B}, \mathrm{C}$ be fuzzy numbers. Then

$$
[f(A, B, C)]_{\alpha}=f\left([A]_{\alpha},[B]_{\alpha},[C]_{\alpha}\right), \quad \alpha \in(0,1] .
$$

Lemma $3.2([2,31])$. Let $u \in R_{f}$, write $[u]_{\alpha}=\left[u_{l, \alpha}, u_{r, \alpha}\right], \alpha \in(0,1]$. Then $u_{l, \alpha}$ and $u_{r, \alpha}$ can be regarded as functions on $(0,1]$ which satisfy

(i) $\mathrm{u}_{\mathrm{l}, \alpha}$ is nondecreasing and left continuous;

(ii) $u_{r, \alpha}$ is nonincreasing and left continuous;

(iii) $u_{l, \alpha} \leqslant u_{r, \alpha}$.

Conversely for any functions $a(\alpha)$ and $b(\alpha)$ defined on $(0,1]$ which satisfy (i)-(iii) in the above, there exists a unique $u \in R_{f}$ such that $u(\alpha)=[a(\alpha), b(\alpha)]$, for any $\alpha \in(0,1]$.

Theorem 3.3. Consider equation (1.1), where A, B, C, D are positive fuzzy numbers. Then for any positive fuzzy numbers $x_{-4}, x_{-3}, x_{-2}, x_{-1}, x_{0}$, there exists a unique positive solution $x_{n}$ of (1.1) with initial conditions $x_{-4}, x_{-3}, x_{-2}, x_{-1}, x_{0}$.

Proof. Suppose that there exists a sequence of fuzzy numbers $\left\{x_{n}\right\}$ satisfying (1.1) with initial conditions $x_{-4}, x_{-3}, x_{-2}, x_{-1}, x_{0}$. Consider the $\alpha$-cuts, $\alpha \in(0,1]$,

$$
\begin{aligned}
& {[A]_{\alpha}=\left[A_{l, \alpha}, A_{r, \alpha}\right],[B]_{\alpha}=\left[B_{l, \alpha}, B_{r, \alpha}\right],[C]_{\alpha}=\left[C_{l, \alpha}, C_{r, \alpha}\right],} \\
& {[D]_{\alpha}=\left[D_{l, \alpha}, D_{r, \alpha}\right],\left[x_{n}\right]_{\alpha}=\left[L_{n, \alpha}, R_{n, \alpha}\right], n=-4,-3, \cdots .}
\end{aligned}
$$

Then from (1.1), (3.1) and Lemma 3.1, it follows that

$$
\begin{aligned}
{\left[x_{n+1}\right]_{\alpha}=\left[L_{n+1, \alpha}, R_{n+1, \alpha}\right] } & =\left[\frac{A x_{n-1} x_{n-2}}{D+B x_{n-3}+C x_{n-4}}\right]_{\alpha}=\frac{\left[A x_{n-1} x_{n-2}\right]_{\alpha}}{\left[D+B x_{n-3}+C x_{n-4}\right]_{\alpha}} \\
& =\frac{\left[A_{l, \alpha}, A_{r, \alpha}\right]\left[L_{n-1, \alpha}, R_{n-1, \alpha}\right]\left[L_{n-2, \alpha}, R_{n-2, \alpha}\right]}{\left[D_{l, \alpha}, D_{r, \alpha}\right]+\left[B_{l, \alpha}, B_{r, \alpha}\right]\left[L_{n-3, \alpha}, R_{n-3, \alpha}\right]+\left[C_{l, \alpha}, C_{r, \alpha}\right]\left[L_{n-4, \alpha}, R_{n-4, \alpha}\right]} \\
& =\frac{\left[A_{l, \alpha} L_{n-1, \alpha} L_{n-2, \alpha}, A_{r, \alpha} R_{n-1, \alpha} R_{n-2, \alpha}\right]}{\left[D_{l, \alpha}+B_{l, \alpha} L_{n-3, \alpha}+C_{l, \alpha} L_{n-4, \alpha}, D_{r, \alpha}+B_{r, \alpha} R_{n-3, \alpha}+C_{r, \alpha} R_{n-4, \alpha}\right]} \\
& =\left[\frac{A_{l, \alpha} L_{n-1, \alpha} L_{n-2, \alpha}}{D_{r, \alpha}+B_{r, \alpha} R_{n-3, \alpha}+C_{r, \alpha} R_{n-4, \alpha}}, \frac{A_{r, \alpha} R_{n-1, \alpha} R_{n-2, \alpha}}{D_{l, \alpha}+B_{l, \alpha} L_{n-3, \alpha}+C_{l, \alpha} L_{n-4, \alpha}}\right],
\end{aligned}
$$


from the above equation, for $\alpha \in(0,1], n=-4,-3, \cdots$, we have

$$
L_{n+1, \alpha}=\frac{A_{l, \alpha} L_{n-1, \alpha} L_{n-2, \alpha}}{D_{r, \alpha}+B_{r, \alpha} R_{n-3, \alpha}+C_{r, \alpha} R_{n-4, \alpha}}, \quad R_{n+1, \alpha}=\frac{A_{r, \alpha} R_{n-1, \alpha} R_{n-2, \alpha}}{D_{l, \alpha}+B_{l, \alpha} L_{n-3, \alpha}+C_{l, \alpha} L_{n-4, \alpha}} .
$$

Then from Lemma 2.3 it is obvious that for any $\left(L_{j, \alpha}, R_{j, \alpha}\right), j=-4,-3,-2,-1,0$, there exists a unique solution $\left(L_{n, \alpha}, R_{n, \alpha}\right)$ of the systems (3.2) with initial conditions $\left(L_{j, \alpha}, R_{j, \alpha}\right), j=-4,-3,-2,-1,0, \alpha \in$ $(0,1]$.

Conversely, we prove that $\left(L_{n, \alpha}, R_{n, \alpha}\right), \alpha \in(0,1]$ where $\left(L_{n, \alpha}, R_{n, \alpha}\right)$ is the solution of the system (3.2) with initial conditions $\left(L_{j, \alpha}, R_{j, \alpha}\right), j=-4,-3,-2,-1,0$ determines the solution $\left\{x_{n}\right\}$ of (1.1) with initial conditions $x_{-4}, x_{-3}, x_{-2}, x_{-1}, x_{0}$ such that

$$
\left[x_{n}\right]_{\alpha}=\left[L_{n, \alpha}, R_{n, \alpha}\right], \quad \alpha \in(0,1], \quad n=-4,-3, \cdots .
$$

From Lemma 3.2 and since $A, B, C, D, x_{j}, j=-4,-3,-2,-1,0$ are positive fuzzy numbers for any $\alpha_{1}, \alpha_{2} \in$ $(0,1], \alpha_{1}<\alpha_{2}$, we have

$$
\begin{array}{ll}
0<A_{l, \alpha_{1}} \leqslant A_{l, \alpha_{2}} \leqslant A_{r, \alpha_{2}} \leqslant A_{r, \alpha_{1}}, & 0<B_{l, \alpha_{1}} \leqslant B_{l, \alpha_{2}} \leqslant B_{r, \alpha_{2}} \leqslant B_{r, \alpha_{1}}, \\
0<C_{l, \alpha_{1}} \leqslant C_{l, \alpha_{2}} \leqslant C_{r, \alpha_{2}} \leqslant C_{r, \alpha_{1}}, & 0<D_{l, \alpha_{1}} \leqslant D_{l, \alpha_{2}} \leqslant D_{r, \alpha_{2}} \leqslant D_{r, \alpha_{1}}, \\
0<L_{j, \alpha_{1}} \leqslant L_{j, \alpha_{2}} \leqslant R_{j, \alpha_{2}} \leqslant R_{j, \alpha_{1}}, & j=-4,-3,-2,-1,0 .
\end{array}
$$

We prove by mathematical induction that

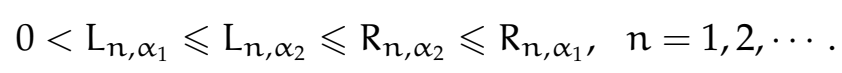

From (3.4), we have that (3.5) holds for $n=-4,-3, \cdots, 0$. Suppose that (3.5) are true for $n \leqslant k, k \in$ $\{1,2, \cdots\}$, then from (3.3), (3.4), (3.5), it follows that for $n=k+1$

$$
\begin{aligned}
L_{K+1, \alpha_{1}} & =\frac{A_{l, \alpha_{1}} L_{K-1, \alpha_{1}} L_{K-2, \alpha_{1}}}{D_{r, \alpha_{1}}+B_{r, \alpha_{1}} R_{K-3, \alpha_{1}}+C_{r, \alpha_{1}} R_{K-4, \alpha_{1}}} \\
& \leqslant \frac{A_{l, \alpha_{2}} L_{K-1, \alpha_{2}} L_{K-2, \alpha_{2}}}{D_{r, \alpha_{2}}+B_{r, \alpha_{2}} R_{K-3, \alpha_{2}}+C_{r, \alpha_{2}} R_{K-4, \alpha_{2}}}=L_{K+1, \alpha_{2}} \\
& \leqslant \frac{A_{r, \alpha_{2}} R_{K-1, \alpha_{2}} R_{K-2, \alpha_{2}}}{D_{l, \alpha_{2}}+B_{l, \alpha_{2}} L_{K-3, \alpha_{2}}+C_{l, \alpha_{2}} L_{K-4, \alpha_{2}}}=R_{K+1, \alpha_{2}} \\
& \leqslant \frac{A_{r, \alpha_{1}} R_{K-1, \alpha_{1}} R_{K-2, \alpha_{1}}}{D_{l, \alpha_{1}}+B_{l, \alpha_{1}} L_{K-3, \alpha_{1}}+C_{l, \alpha_{1}} L_{K-4, \alpha_{1}}}=R_{K+1, \alpha_{1} .} .
\end{aligned}
$$

Therefore (3.5) are true.

Moreover from (3.2), we have

$$
L_{1, \alpha}=\frac{A_{l, \alpha} L_{-1, \alpha} L_{-2, \alpha}}{D_{r, \alpha}+B_{r, \alpha} R_{-3, \alpha}+C_{r, \alpha} R_{-4, \alpha}}, \quad R_{1, \alpha}=\frac{A_{r, \alpha} R_{-1, \alpha} R_{-2, \alpha}}{D_{l, \alpha}+B_{l, \alpha} L_{-3, \alpha}+C_{l, \alpha} L_{-4, \alpha}}, \alpha \in(0,1] .
$$

Then since $A, B, C, D, x_{j}, j=-4,-3,-2,-1,0$ are positive fuzzy numbers, from Lemma 3.2, we have that $A_{l, \alpha}, A_{r, \alpha}, B_{l, \alpha}, B_{r, \alpha}, C_{l, \alpha}, C_{r, \alpha}, D_{l, \alpha}, D_{r, \alpha}, L_{-1, \alpha}, R_{-1, \alpha}, L_{-2, \alpha}, R_{-2, \alpha}, L_{-3, \alpha}, R_{-3, \alpha}, L_{-4, \alpha}, R_{-4, \alpha}$ are left continuous. Thus, from (3.6) we have that $L_{1, \alpha}, R_{1, \alpha}$ are also left continuous. Moreover, we can prove that $L_{n, \alpha}, R_{n, \alpha}, n=1,2, \cdots$, are left continuous by mathematical induction.

Now, we prove that the support of $x_{n}$, Supp $x_{n}=\overline{\bigcup_{\alpha \in(0,1]}\left[L_{n, \alpha}, R_{n, \alpha}\right]}$ is compact. It is sufficient to prove that $\bigcup_{\alpha \in(0,1]}\left[L_{n, \alpha}, R_{n, \alpha}\right]$ is bounded.

Let $n=1$. Since $A, B, C, D, x_{j}, j=-4,-3,-2,-1,0$ are positive fuzzy numbers, there exist constants 
$M_{i}, N_{i}>0, i=1,2,3,4$ such that for all $\alpha \in(0,1]$

$$
\begin{array}{ll}
{\left[A_{l, \alpha}, A_{r, \alpha}\right] \subset\left[M_{1}, N_{1}\right],} & {\left[B_{l, \alpha}, B_{r, \alpha}\right] \subset\left[M_{2}, N_{2}\right],} \\
{\left[C_{l, \alpha}, C_{r, \alpha}\right] \subset\left[M_{3}, N_{3}\right],} & {\left[D_{l, \alpha}, D_{r, \alpha}\right] \subset\left[M_{4}, N_{4}\right],} \\
{\left[L_{j, \alpha}, R_{j, \alpha}\right] \subset\left[M_{j}, N_{j}\right],} & j=-4,-3,-2,-1,0,
\end{array}
$$

therefore from (3.6) and (3.7) we can prove that

$$
\left[L_{1, \alpha}, R_{1, \alpha}\right] \subset\left[\frac{M_{1} M_{-1} M_{-2}}{N_{4}+N_{2} N_{-3}+N_{3} N_{-4}}, \frac{N_{1} N_{-1} N_{-2}}{M_{4}+M_{2} M_{-3}+M_{3} M_{-4}}\right], \alpha \in(0,1],
$$

from which it is obvious that

$$
\bigcup_{\alpha \in(0,1]}\left[\mathrm{L}_{1, \alpha}, \mathrm{R}_{1, \alpha}\right] \subset\left[\frac{\mathrm{M}_{1} \mathrm{M}_{-1} \mathrm{M}_{-2}}{\mathrm{~N}_{4}+\mathrm{N}_{2} \mathrm{~N}_{-3}+\mathrm{N}_{3} \mathrm{~N}_{-4}}, \frac{\mathrm{N}_{1} \mathrm{~N}_{-1} \mathrm{~N}_{-2}}{\mathrm{M}_{4}+\mathrm{M}_{2} \mathrm{M}_{-3}+\mathrm{M}_{3} \mathrm{M}_{-4}}\right]
$$

Relation (3.8) implies that $\overline{\bigcup_{\alpha \in(0,1]}\left[\mathrm{L}_{1, \alpha}, \mathrm{R}_{1, \alpha}\right]}$ is compact and $\overline{\bigcup_{\alpha \in(0,1]}\left[\mathrm{L}_{1, \alpha}, \mathrm{R}_{1, \alpha}\right]} \subset(0, \infty)$. Thus, by mathematical induction we can prove that $\overline{\bigcup_{\alpha \in(0,1]}\left[L_{n, \alpha}, R_{n, \alpha}\right]}$ is compact and

$$
\overline{\bigcup_{\alpha \in(0,1]}\left[L_{n, \alpha}, R_{n, \alpha}\right]} \subset(0, \infty), n=1,2, \cdots .
$$

Therefore from Lemma 3.2, relations (3.5) and (3.9), and $L_{n, \alpha}, R_{n, \alpha}$ are left continuous, we have that $\left[L_{n, \alpha}, R_{n, \alpha}\right]$ determines a sequence of positive fuzzy numbers $\left\{x_{n}\right\}$ such that (1.1) holds.

Now, we prove that $\left\{x_{n}\right\}$ is the solution of (1.1) with initial dates $x_{-4}, x_{-3}, x_{-2}, x_{-1}, x_{0}$. Since for all

$$
\begin{aligned}
{\left[x_{n+1}\right]_{\alpha} } & =\left[L_{n+1, \alpha}, R_{n+1, \alpha}\right] \\
& =\left[\frac{A_{l, \alpha} L_{n-1, \alpha} L_{n-2, \alpha}}{D_{r, \alpha}+B_{r, \alpha} R_{n-3, \alpha}+C_{r, \alpha} R_{n-4, \alpha}}, \frac{A_{r, \alpha} R_{n-1, \alpha} R_{n-2, \alpha}}{D_{l, \alpha}+B_{l, \alpha} L_{n-3, \alpha}+C_{l, \alpha} L_{n-4, \alpha}}\right] \\
& =\left[\frac{A x_{n-1} x_{n-2}}{D+B x_{n-3}+C x_{n-4}}\right]_{\alpha}{ }^{\prime}
\end{aligned}
$$

we have that $\left\{x_{n}\right\}$ is the solution of (1.1) with initial dates $x_{-4}, x_{-3}, x_{-2}, x_{-1}, x_{0}$.

Suppose that there exists another solution $\left\{x_{n}^{*}\right\}$ of (1.1) with initial conditions $x_{-4}, x_{-3}, x_{-2}, x_{-1}, x_{0}$, then we can easily prove by arguing as above that

$$
\left[x_{n}^{*}\right]_{\alpha}=\left[L_{n, \alpha}, R_{n, \alpha}\right], \quad \alpha \in(0,1], \quad n=0,1, \cdots,
$$

then from (3.3) and (3.10) we have that

$$
\left[x_{n}\right]_{\alpha}=\left[x_{n}^{*}\right]_{\alpha,} \alpha \in(0,1], \quad n=-4,-3, \cdots,
$$

from which it holds $x_{n}=x_{n}^{*}, \alpha \in(0,1], n=-4,-3, \cdots$, and then the proof is completed.

In the following theorem we investigate the asymptotic behavior of the equilibrium point of (1.1).

If $\left\{x_{n}\right\}$ is the unique positive solution of (1.1) with the initial values $x_{-4}, x_{-3}, x_{-2}, x_{-1}, x_{0}$ such that

$$
\left[x_{n}\right]_{\alpha}=\left[L_{n, \alpha}, R_{n, \alpha}\right], \quad \alpha \in(0,1], \quad n=0,1, \cdots,
$$

then we obtain that $\left(L_{n, \alpha}, R_{n, \alpha}\right)$ satisfies the family of systems of ordinary difference equations

$$
\begin{aligned}
L_{n+1, \alpha} & =\frac{A_{l, \alpha} L_{n-1, \alpha} L_{n-2, \alpha}}{D_{r, \alpha}+B_{r, \alpha} R_{n-3, \alpha}+C_{r, \alpha} R_{n-4, \alpha}}, \\
R_{n+1, \alpha} & =\frac{A_{r, \alpha} R_{n-1, \alpha} R_{n-2, \alpha}}{D_{l, \alpha}+B_{l, \alpha} L_{n-3, \alpha}+C_{l, \alpha} L_{n-4, \alpha}}, \quad \alpha \in(0,1], \quad n=0,1, \cdots .
\end{aligned}
$$


In order to study the asymptotic behavior of (1.1), from (3.11), we will consider the following systems of ordinary parametric difference equations

$$
\begin{aligned}
& y_{n+1}=\frac{a y_{n-1} y_{n-2}}{h+d z_{n-3}+f z_{n-4}}, \\
& z_{n+1}=\frac{b z_{n-1} z_{n-2}}{g+c y_{n-3}+e y_{n-4}}, \quad n=0,1, \cdots,
\end{aligned}
$$

where the parametric $a, b, c, d, e, f, g, h$ are positive real constants and initial values $y_{-4}, y_{-3}, y_{-2}, y_{-1}, y_{0}$, $z_{-4}, z_{-3}, z_{-2}, z_{-1}, z_{0}$ are also positive real constants. From Lemma 2.3, we know that the systems of ordinary parametric difference equations (3.12) have a unique solutions $\left(y_{n}, z_{n}\right)$ for any initial values. Moreover, we can easily obtain that the systems (3.12) have three equilibrium points

$$
\bar{x}_{1}=\left(\bar{y}_{1}, \bar{z}_{1}\right)=(0,0), \quad \bar{x}_{2}=\left(\bar{y}_{2}, \bar{z}_{2}\right)=\left(0, \frac{g}{b}\right), \quad \bar{x}_{3}=\left(\bar{y}_{3}, \bar{z}_{3}\right)=\left(\frac{h}{a}, 0\right),
$$

if $a b>(c+e)(d+f)$, equation (3.12) has the fourth positive equilibrium point $\bar{X}_{4}$,

$$
\bar{X}_{4}=\left(\bar{y}_{4}, \bar{z}_{4}\right)=\left(\frac{b h+g(d+f)}{a b-(c+e)(d+f)}, \frac{a g+h(c+e)}{a b-(c+e)(d+f)}\right) .
$$

Theorem 3.4. The equilibrium point $\overline{\mathrm{X}}_{1}$ of (3.12) is locally asymptotically stable.

Proof. Let $\mathrm{F}:\left(\mathrm{R}^{+}\right)^{4} \rightarrow \mathrm{R}^{+}, \mathrm{H}:\left(\mathrm{R}^{+}\right)^{4} \rightarrow \mathrm{R}^{+}$be multivariate function defined by

$$
\mathrm{F}\left(y_{n-1}, y_{n-2}, z_{n-3}, z_{n-4}\right)=\frac{a y_{n-1} y_{n-2}}{h+d z_{n-3}+f z_{n-4}}, \quad H\left(z_{n-1}, z_{n-2}, y_{n-3}, y_{n-4}\right)=\frac{b z_{n-1} z_{n-2}}{g+c y_{n-3}+e y_{n-4}},
$$

thus, we have

$$
\begin{array}{ll}
\mathrm{F}_{y_{n-1}}=\frac{a y_{n-2}}{h+d z_{n-3}+f z_{n-4}}, & F_{y_{n-2}}=\frac{a y_{n-1}}{h+d z_{n-3}+f z_{n-4}}, \\
F_{z_{n-3}}=-\frac{a d y_{n-1} y_{n-2}}{\left(h+d z_{n-3}+f z_{n-4}\right)^{2}}, & F_{z_{n-4}}=-\frac{a f y_{n-1} y_{n-2}}{\left(h+d z_{n-3}+f z_{n-4}\right)^{2}}, \\
H_{z_{n-1}}=\frac{b z_{n-2}}{g+c y_{n-3}+e y_{n-4}}, & \mathrm{H}_{z_{n-2}}=\frac{b z_{n-1}}{g+c y_{n-3}+e y_{n-4}}, \\
H_{y_{n-3}}=-\frac{b c z_{n-1} z_{n-2}}{\left(g+c y_{n-3}+e y_{n-4}\right)^{2}}, & H_{y_{n-4}}=-\frac{b e z_{n-1} z_{n-2}}{\left(g+c y_{n-3}+e y_{n-4}\right)^{2}} .
\end{array}
$$

Moreover, we can easily obtain that the linearized equations of the system (3.12) about the equilibrium point $\bar{X}_{1}$ is

$$
\varphi_{\mathrm{n}+1}=\mathrm{D}_{1} \varphi_{\mathrm{n}}
$$

where

$$
\phi_{n}=\left[\begin{array}{l}
y_{n} \\
y_{n-1} \\
y_{n-2} \\
y_{n-3} \\
y_{n-4} \\
z_{n} \\
z_{n-1} \\
z_{n-2} \\
z_{n-3} \\
z_{n-4}
\end{array}\right], \quad D_{1}=\left[\begin{array}{llllllllll}
0 & 0 & 0 & 0 & 0 & 0 & 0 & 0 & 0 & 0 \\
1 & 0 & 0 & 0 & 0 & 0 & 0 & 0 & 0 & 0 \\
0 & 1 & 0 & 0 & 0 & 0 & 0 & 0 & 0 & 0 \\
0 & 0 & 1 & 0 & 0 & 0 & 0 & 0 & 0 & 0 \\
0 & 0 & 0 & 1 & 0 & 0 & 0 & 0 & 0 & 0 \\
0 & 0 & 0 & 0 & 0 & 0 & 0 & 0 & 0 & 0 \\
0 & 0 & 0 & 0 & 0 & 1 & 0 & 0 & 0 & 0 \\
0 & 0 & 0 & 0 & 0 & 0 & 1 & 0 & 0 & 0 \\
0 & 0 & 0 & 0 & 0 & 0 & 0 & 1 & 0 & 0 \\
0 & 0 & 0 & 0 & 0 & 0 & 0 & 0 & 1 & 0
\end{array}\right],
$$


the characteristic equation with (3.14) is $\lambda^{10}=0$, since we have $|\lambda|<1$, from Lemma 2.8, we have that the equilibrium point $\bar{X}_{1}$ of (3.12) is locally asymptotically stable, and then the proof is completed.

Theorem 3.5. The equilibrium point $\bar{X}_{2}$ of (3.12) is unstable.

Proof. From (3.13), we have that the linearized equations of (3.12) about the equilibrium point $\bar{X}_{2}$ is

$$
\varphi_{\mathrm{n}+1}=\mathrm{D}_{2} \varphi_{\mathrm{n}}
$$

where

$$
\Phi_{n}=\left[\begin{array}{l}
y_{n} \\
y_{n-1} \\
y_{n-2} \\
y_{n-3} \\
y_{n-4} \\
z_{n} \\
z_{n-1} \\
z_{n-2} \\
z_{n-3} \\
z_{n-4}
\end{array}\right], \quad D_{2}=\left[\begin{array}{cccccccccc}
0 & 0 & 0 & 0 & 0 & 0 & 0 & 0 & 0 & 0 \\
1 & 0 & 0 & 0 & 0 & 0 & 0 & 0 & 0 & 0 \\
0 & 1 & 0 & 0 & 0 & 0 & 0 & 0 & 0 & 0 \\
0 & 0 & 1 & 0 & 0 & 0 & 0 & 0 & 0 & 0 \\
0 & 0 & 0 & 1 & 0 & 0 & 0 & 0 & 0 & 0 \\
0 & 0 & 0 & -\frac{c}{b} & -\frac{e}{b} & 0 & 1 & 1 & 0 & 0 \\
0 & 0 & 0 & 0 & 0 & 1 & 0 & 0 & 0 & 0 \\
0 & 0 & 0 & 0 & 0 & 0 & 1 & 0 & 0 & 0 \\
0 & 0 & 0 & 0 & 0 & 0 & 0 & 1 & 0 & 0 \\
0 & 0 & 0 & 0 & 0 & 0 & 0 & 0 & 1 & 0
\end{array}\right],
$$

the characteristic equation of the systems (3.15) is

$$
\lambda^{7}\left(\lambda^{3}-\lambda-1\right)=0 .
$$

It is obvious that there exists $|\lambda|>1$ so that $\lambda^{7}\left(\lambda^{3}-\lambda-1\right)=0$, therefore, one of the roots of characteristic equation (3.16) lies outside unit disk, according to Lemma 2.8, we have that the equilibrium point $\bar{X}_{2}$ of (3.12) is unstable, and then the proof is completed.

Theorem 3.6. The equilibrium point $\overline{\mathrm{X}}_{3}$ of (3.12) is unstable.

Proof. From (3.13), we have that the linearized equation of (3.12) about the equilibrium point $\bar{X}_{3}$ is

$$
\varphi_{\mathrm{n}+1}=\mathrm{D}_{3} \varphi_{\mathrm{n}}
$$

where

$$
\phi_{\mathfrak{n}}=\left[\begin{array}{l}
y_{\mathfrak{n}} \\
y_{\mathfrak{n}-1} \\
y_{\mathfrak{n}-2} \\
y_{\mathfrak{n}-3} \\
y_{\mathfrak{n}-4} \\
z_{\mathfrak{n}} \\
z_{n-1} \\
z_{n-2} \\
z_{n-3} \\
z_{n-4}
\end{array}\right], \quad D_{3}=\left[\begin{array}{cccccccccc}
0 & 1 & 1 & 0 & 0 & 0 & 0 & 0 & -\frac{d}{a} & -\frac{f}{a} \\
1 & 0 & 0 & 0 & 0 & 0 & 0 & 0 & 0 & 0 \\
0 & 1 & 0 & 0 & 0 & 0 & 0 & 0 & 0 & 0 \\
0 & 0 & 1 & 0 & 0 & 0 & 0 & 0 & 0 & 0 \\
0 & 0 & 0 & 1 & 0 & 0 & 0 & 0 & 0 & 0 \\
0 & 0 & 0 & 0 & 0 & 0 & 0 & 0 & 0 & 0 \\
0 & 0 & 0 & 0 & 0 & 1 & 0 & 0 & 0 & 0 \\
0 & 0 & 0 & 0 & 0 & 0 & 1 & 0 & 0 & 0 \\
0 & 0 & 0 & 0 & 0 & 0 & 0 & 1 & 0 & 0 \\
0 & 0 & 0 & 0 & 0 & 0 & 0 & 0 & 1 & 0
\end{array}\right],
$$

the characteristic equation of the systems (3.17) is

$$
\lambda^{7}\left(\lambda^{3}-\lambda-1\right)=0,
$$

which is the same with (3.16), therefore the equilibrium point $\bar{X}_{3}$ of (3.12) is unstable, and then the proof is completed. 
Theorem 3.7. If $\mathrm{ab}>(\mathrm{c}+\mathrm{e})(\mathrm{d}+\mathrm{f})$, equation (3.12) has the positive equilibrium point $\overline{\mathrm{X}}_{4}$, and the equilibrium point is unstable.

Proof. From (3.13), we have that the linearized equation of (3.12) about the equilibrium point $\bar{X}_{4}$ is

$$
\varphi_{\mathrm{n}+1}=\mathrm{D}_{4} \varphi_{\mathrm{n}}
$$

where

$$
\phi_{n}=\left[\begin{array}{l}
y_{n} \\
y_{n-1} \\
y_{n-2} \\
y_{n-3} \\
y_{n-4} \\
z_{n} \\
z_{n-1} \\
z_{n-2} \\
z_{n-3} \\
z_{n-4}
\end{array}\right], \quad D_{4}=\left[\begin{array}{cccccccccc}
0 & 1 & 1 & 0 & 0 & 0 & 0 & 0 & -\frac{d}{a} & -\frac{f}{a} \\
1 & 0 & 0 & 0 & 0 & 0 & 0 & 0 & 0 & 0 \\
0 & 1 & 0 & 0 & 0 & 0 & 0 & 0 & 0 & 0 \\
0 & 0 & 1 & 0 & 0 & 0 & 0 & 0 & 0 & 0 \\
0 & 0 & 0 & 1 & 0 & 0 & 0 & 0 & 0 & 0 \\
0 & 0 & 0 & -\frac{c}{b} & -\frac{e}{b} & 0 & 1 & 1 & 0 & 0 \\
0 & 0 & 0 & 0 & 0 & 1 & 0 & 0 & 0 & 0 \\
0 & 0 & 0 & 0 & 0 & 0 & 1 & 0 & 0 & 0 \\
0 & 0 & 0 & 0 & 0 & 0 & 0 & 1 & 0 & 0 \\
0 & 0 & 0 & 0 & 0 & 0 & 0 & 0 & 1 & 0
\end{array}\right],
$$

the characteristic equation of the systems (3.18) is

$$
\lambda^{10}-2 \lambda^{8}-2 \lambda^{7}+\lambda^{6}+2 \lambda^{5}+\lambda^{4}-\frac{c d}{a b} \lambda^{2}-\left(\frac{c f+d e}{a b}\right) \lambda-\frac{e f}{a b}=0,
$$

from (3.19), we have

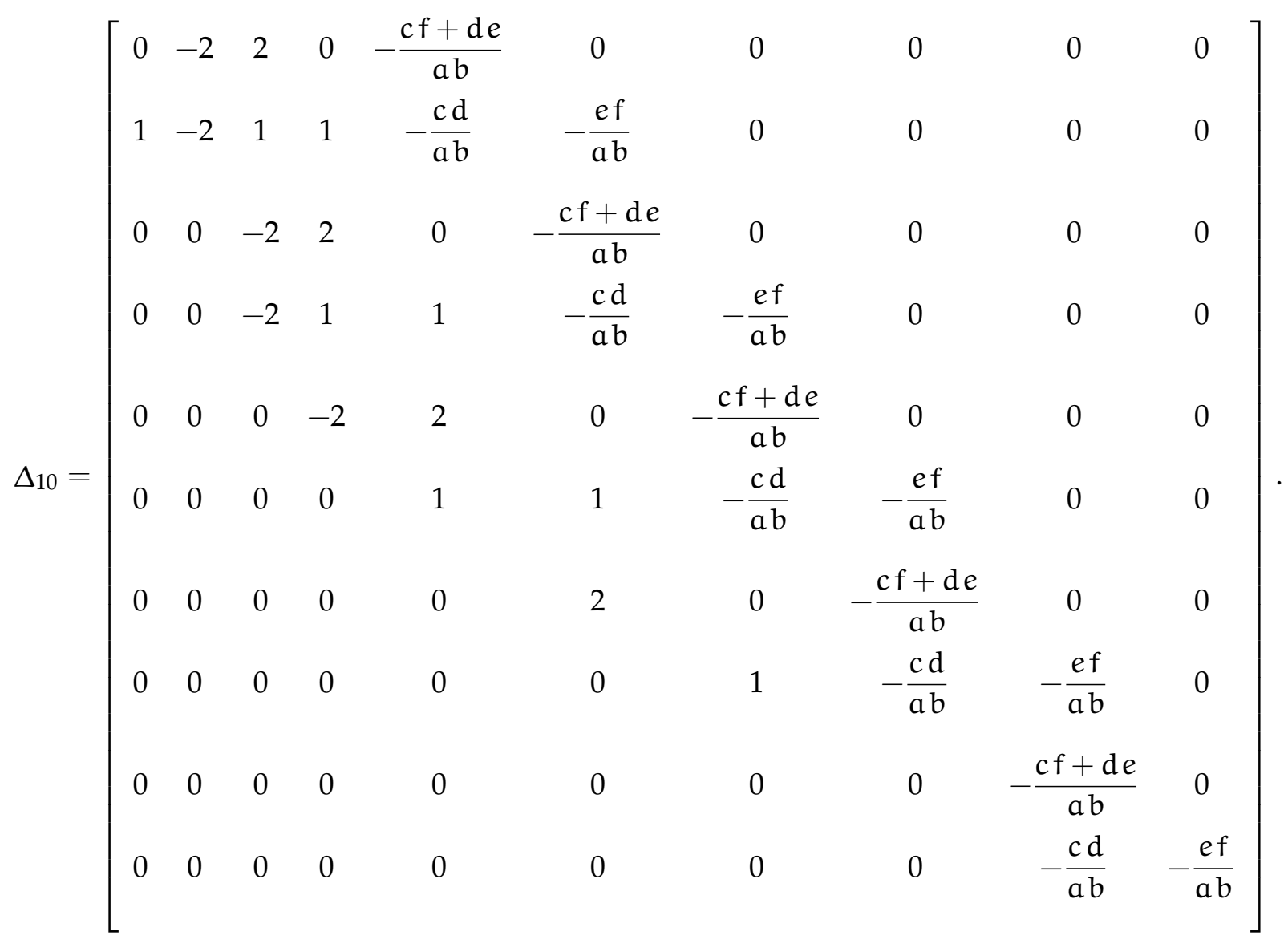

We can see that not all $\Delta_{k}>0, k=1,2, \cdots, 10$, from Lemma 2.8 and Lemma 2.9, we obtain that the equilibrium point $\bar{X}_{4}$ is unstable, and then the proof is completed. 
Theorem 3.8. Let $\mathrm{I}_{x}, \mathrm{I}_{\mathrm{y}}$ be some intervals of real numbers and assume that $\mathrm{f}: \mathrm{I}_{x}^{\mathrm{k}+1} \times \mathrm{I}_{\mathrm{y}}^{\mathrm{l}+1} \rightarrow \mathrm{I}_{x}$ and $\mathrm{g}$ : $\mathrm{I}_{\mathrm{x}}^{\mathrm{k}+1} \times \mathrm{I}_{y}^{\mathrm{l}+1} \rightarrow \mathrm{I}_{y}$ be continuously differentiable functions satisfying mixed monotone property. If there exists

$$
\left\{\begin{array}{l}
\mathrm{m}_{0} \leqslant \min \left\{x_{-k}, \cdots, x_{0}, y_{-l}, \cdots, y_{0}\right\} \leqslant \max \left\{x_{-k}, \cdots, x_{0}, y_{-l}, \cdots, y_{0}\right\} \leqslant M_{0} \\
n_{0} \leqslant \min \left\{x_{-k}, \cdots, x_{0}, y_{-l}, \cdots, y_{0}\right\} \leqslant \max \left\{x_{-k}, \cdots, x_{0}, y_{-l}, \cdots, y_{0}\right\} \leqslant N_{0}
\end{array}\right.
$$

such that

$$
\left\{\begin{array}{l}
m_{0} \leqslant f\left(\left[m_{0}\right]_{p},\left[M_{0}\right]_{q},\left[n_{0}\right]_{s},\left[N_{0}\right]_{t}\right) \leqslant f\left(\left[M_{0}\right]_{p},\left[m_{0}\right]_{q},\left[N_{0}\right]_{s},\left[n_{0}\right]_{t}\right) \leqslant M_{0} \\
n_{0} \leqslant g\left(\left[m_{0}\right]_{p_{1}},\left[M_{0}\right]_{q_{1}},\left[n_{0}\right]_{s_{1}},\left[N_{0}\right]_{t_{1}}\right) \leqslant g\left(\left[M_{0}\right]_{p_{1}},\left[m_{0}\right]_{q_{1}},\left[N_{0}\right]_{s_{1}},\left[n_{0}\right]_{t_{1}}\right) \leqslant N_{0}
\end{array}\right.
$$

then there exist $(m, M) \in\left[m_{0}, M_{0}\right]^{2}$ and $(n, N) \in\left[n_{0}, N_{0}\right]^{2}$ satisfying

$$
\left\{\begin{array}{l}
M=f\left([M]_{p},[m]_{q},[N]_{s},[n]_{t}\right), \quad m=f\left([m]_{p},[M]_{q},[n]_{s},[N]_{t}\right) \\
N=g\left([M]_{p_{1}},[m]_{q_{1}},[N]_{s_{1}},[n]_{t_{1}}\right), \quad n=g\left([m]_{p_{1}},[M]_{q_{1}},[n]_{s_{1}},[N]_{t_{1}}\right) .
\end{array}\right.
$$

Moreover, if $\mathrm{m}=\mathrm{M}, \mathrm{n}=\mathrm{N}$, then (2.1) has a unique equilibrium point $(\overline{\mathrm{x}}, \overline{\mathrm{y}}) \in\left[\mathrm{m}_{0}, \mathrm{M}_{0}\right] \times\left[\mathrm{n}_{0}, \mathrm{~N}_{0}\right]$ and every solution of $(2.1)$ converges to $(\bar{x}, \bar{y})$.

Proof. Using $m_{0}, M_{0}, n_{0}$ and $N_{0}$ as two couples of initial iterations, we construct four sequences $\left\{m_{i}\right\},\left\{M_{i}\right\}$, $\left\{n_{i}\right\}$ and $\left\{N_{i}\right\}(i=1,2, \cdots)$ from the following equations

$$
\left\{\begin{array}{l}
m_{i}=f\left(\left[m_{i-1}\right]_{p},\left[M_{i-1}\right]_{q},\left[n_{i-1}\right]_{s},\left[N_{i-1}\right]_{t}\right), \quad M_{i}=f\left(\left[M_{i-1}\right]_{p},\left[m_{i-1}\right]_{q},\left[N_{i-1}\right]_{s},\left[n_{i-1}\right]_{t}\right), \\
n_{i}=g\left(\left[m_{i-1}\right]_{p_{1}},\left[M_{i-1}\right]_{q_{1}},\left[n_{i-1}\right]_{s_{1}},\left[N_{i-1}\right]_{t_{1}}\right), \quad N_{i}=g\left(\left[M_{i-1}\right]_{p_{1}},\left[m_{i-1}\right]_{q_{1}},\left[N_{i-1}\right]_{s_{1}},\left[n_{i-1}\right]_{t_{1}}\right) .
\end{array}\right.
$$

It is obvious from the mixed monotone property of $f$ and $g$ that the sequences $\left\{m_{i}\right\},\left\{M_{i}\right\},\left\{n_{i}\right\}$ and $\left\{N_{i}\right\}$, possess the following monotone property

$$
\left\{\begin{array}{l}
m_{0} \leqslant m_{1} \leqslant \cdots \leqslant m_{i} \leqslant \cdots \leqslant M_{i} \leqslant \cdots \leqslant M_{1} \leqslant M_{0} \\
n_{0} \leqslant n_{1} \leqslant \cdots \leqslant n_{i} \leqslant \cdots \leqslant N_{i} \leqslant \cdots \leqslant N_{1} \leqslant N_{0}
\end{array}\right.
$$

where $i=0,1,2, \cdots$, and

$$
m_{i} \leqslant x_{u} \leqslant M_{i}, \quad n_{i} \leqslant y_{v} \leqslant N_{i}, \text { for } u \geqslant(k+1) i+1, \quad v \geqslant(l+1) i+1, i=0,1,2, \cdots .
$$

Set

$$
m=\lim _{i \rightarrow \infty} m_{i}, M=\lim _{i \rightarrow \infty} M_{i}, \quad n=\lim _{i \rightarrow \infty} n_{i}, N=\lim _{i \rightarrow \infty} N_{i}
$$

Then

$$
m \leqslant \lim _{i \rightarrow \infty} \inf x_{i} \leqslant \lim _{i \rightarrow \infty} \sup x_{i} \leqslant M, \quad n \leqslant \lim _{i \rightarrow \infty} \inf y_{i} \leqslant \lim _{i \rightarrow \infty} \sup y_{i} \leqslant N .
$$

By the continuity of $f$ and $g$, one has

$$
\left\{\begin{array}{l}
M=f\left([M]_{p},[m]_{q},[N]_{s},[n]_{t}\right), \quad m=f\left([m]_{p},[M]_{q},[n]_{s},[N]_{t}\right), \\
N=g\left([M]_{p_{1}},[m]_{q_{1}},[N]_{s_{1}},[n]_{t_{1}}\right), \quad n=g\left([m]_{p_{1}},[M]_{q_{1}},[n]_{s_{1}},[N]_{t_{1}}\right) .
\end{array}\right.
$$

Moreover, if $m=M, n=N$, then $m=M=\lim _{i \rightarrow \infty} x_{i}=\bar{x}, n=N=\lim _{i \rightarrow \infty} y_{i}=\bar{y}$, and then the proof is completed.

Theorem 3.9. If $\mathrm{a}=\mathrm{b}, \mathrm{h}=\mathrm{g}, \mathrm{c}=\mathrm{d}, \mathrm{e}=\mathrm{f}$, then the equilibrium point $(0,0)$ of the system (3.12) is global attractor for any conditions $\left(y_{-i}, z_{-i}\right) \in\left(0, \frac{h}{2 a}\right) \times\left(0, \frac{h}{2 a}\right), i=-4,,-3, \cdots, 0$. 
Proof. Since $a=b, h=g, c=d, e=f$, hence the system (3.12) is changed to

$$
\begin{aligned}
& y_{n+1}=\frac{a y_{n-1} y_{n-2}}{h+d z_{n-3}+f z_{n-4}}, \\
& z_{n+1}=\frac{a z_{n-1} z_{n-2}}{h+d y_{n-3}+f y_{n-4}}, n=0,1, \cdots .
\end{aligned}
$$

Let $(f, g):\left(0, \frac{h}{2 a}\right)^{10} \times\left(0, \frac{h}{2 a}\right)^{10} \rightarrow(0, \infty) \times(0, \infty)$ be a function defined by

$$
\begin{aligned}
& f\left(y_{n}, y_{n-1}, y_{n-2}, y_{n-3}, y_{n-4}, z_{n}, z_{n-1}, z_{n-2}, z_{n-3}, z_{-4}\right)=\frac{a y_{n-1} y_{n-2}}{h+d z_{n-3}+f z_{n-4}}, \\
& g\left(y_{n}, y_{n-1}, y_{n-2}, y_{n-3}, y_{n-4}, z_{n}, z_{n-1}, z_{n-2}, z_{n-3}, z_{-4}\right)=\frac{a z_{n-1} z_{n-2}}{h+d y_{n-3}+f y_{n-4}} .
\end{aligned}
$$

Set

we can obtain that

$$
f=\frac{a u v}{h+d w+f s}, \quad g=\frac{a u^{*} v^{*}}{h+d w^{*}+f s^{*}},
$$

$$
\begin{array}{rlrl}
f_{u} & =\frac{a v}{h+d w+f s}>0, & f_{v} & =\frac{a u}{h+d w+f s}>0, \\
f_{w} & =-\frac{a d u v}{(h+d w+f s)^{2}}<0, & f_{s}=-\frac{a f u v}{(h+d w+f s)^{2}}<0, \\
g_{u^{*}}=\frac{a v^{*}}{h+d w^{*}+f s^{*}}>0, & g_{v^{*}}=\frac{a u^{*}}{h+d w^{*}+f s^{*}}>0, \\
g_{w^{*}}=-\frac{a d u^{*} v^{*}}{\left(h+d w^{*}+f s^{*}\right)^{2}}<0, & g_{s^{*}}=-\frac{a f u^{*} v^{*}}{\left(h+d w^{*}+f s^{*}\right)^{2}}<0,
\end{array}
$$

which implies that $f$ and $g$ possess a mixed monotone property.

Let

$$
M_{0}=N_{0}=\max \left\{y_{-4}, y_{-3}, \cdots, y_{0}, z_{-4}, z_{-3}, \cdots, z_{0}\right\}, \quad \frac{a M_{0}-h}{d+f}<m_{0}=n_{0}<0,
$$

we have

$$
m_{0} \leqslant \frac{a m_{0}^{2}}{h+d N_{0}+f N_{0}} \leqslant \frac{a M_{0}^{2}}{h+d n_{0}+f n_{0}} \leqslant M_{0}, \quad n_{0} \leqslant \frac{a n_{0}^{2}}{h+d M_{0}+f M_{0}} \leqslant \frac{a N_{0}^{2}}{h+d m_{0}+f m_{0}} \leqslant N_{0} .
$$

It is obvious that $m_{i}=n_{i}, M_{i}=N_{i}, i=0,1, \cdots$, then from the system (3.12) and Theorem 3.8, there exist $m, M \in\left[m_{0}, M_{0}\right], n=m, N=M$, satisfying

$$
m=\frac{a m^{2}}{h+d N+f N}, \quad n=\frac{a n^{2}}{h+d M+f M}, \quad M=\frac{a M^{2}}{h+d n+f n}, \quad N=\frac{a N^{2}}{h+d m+f m},
$$

thus

$$
[h-a(m+M)](m-M)=0 .
$$

In view of $2 a M_{0}<h$, we have $h-a(m+M)>0$. Then

$$
\mathrm{M}=\mathrm{m}, \quad \mathrm{N}=\mathrm{n} .
$$

It follows by Theorem 3.8 that the equilibrium point $(0,0)$ of the system (3.12) is global attractor. The proof is therefore completed.

Next, we develop stability results for the fuzzy difference equation (1.1) in terms of the stability of the trivial solution of the ordinary difference equations (3.12). For that purpose we introduce the following notion of stability for equation (1.1). It is obvious that (1.1) has the trivial solution $\hat{0}$. 
Definition 3.10 ([16]). The trivial solution $x=\hat{0}$ of (1.1) is said to be

(i) stable, if given $\varepsilon>0$, there exists a $\delta(\varepsilon)>0$ with $\mathrm{D}\left(x_{i}, \hat{0}\right)<\delta, i=-4,-3, \cdots, 0$, implies $\mathrm{D}\left(x_{n}, \hat{0}\right)<$ $\varepsilon$, for any $n>0$, such that for any $x_{i} \in D_{\delta}, i=-4,-3, \cdots, 0$ the solution $x_{n} \in D_{\varepsilon}, n>0$;

(ii) attractive, if there is a $\delta>0$ such that $\mathrm{D}\left(x_{i}, \hat{0}\right)<\delta, i=-4,-3, \cdots, 0$, one has

$$
\lim _{n \rightarrow \infty} \mathrm{D}\left(x_{n}, \hat{0}\right)=0
$$

(iii) asymptotically stable, if (i) and (ii) hold simultaneously.

Theorem 3.11. If the parameters $A, B, C, D$ are positive trivial fuzzy numbers, i.e., positive real numbers, and the initial conditions are positive fuzzy numbers with $\left[x_{i}\right]_{\alpha} \subset(0, D / 2 A), i=-4,-3, \cdots, 0, \alpha \in(0,1]$ then the trivial solution $\mathrm{x}=\hat{0}$ of (1.1) is asymptotically stable with respect to $\mathrm{D}$ as $\mathrm{n} \rightarrow \infty$.

Proof. The result follows from Theorem 3.4 and Theorem 3.9.

\section{Numerical simulation}

In this section some numerical examples are given in order to confirm the results of the previous sections and support our theoretical discussions. The example represents the asymptotically behavior of solutions for the fuzzy difference system (1.1).

Example 4.1. Consider the following fuzzy difference equation

$$
x_{n+1}=\frac{A x_{n-1} x_{n-2}}{D+B x_{n-3}+C x_{n-4}}, \quad n=0,1,2, \cdots,
$$

where $A, B, C, D$ are positive trivial fuzzy numbers. By Theorem 3.11, we take $[A]_{\alpha}=[A, A]=0.3,[B]_{\alpha}=$ $[\mathrm{B}, \mathrm{B}]=3,[\mathrm{C}]_{\alpha}=[\mathrm{C}, \mathrm{C}]=6,[\mathrm{D}]_{\alpha}=[\mathrm{D}, \mathrm{D}]=12, \alpha \in(0,1]$. In addition, from Theorem 3.11, we denote the initial conditions $x_{-4}, x_{-3}, x_{-2}, x_{-1}, x_{0}$ with $\left[x_{i}\right]_{\alpha} \subset(0, D / 2 A), i=-4,-3, \cdots, 0, \alpha \in(0,1]$ such that

$$
\begin{gathered}
x_{0}(x)= \begin{cases}\frac{1}{5} x-1, & 5 \leqslant x \leqslant 10, \\
-\frac{1}{4} x+\frac{7}{2}, & 10 \leqslant x \leqslant 14,\end{cases} \\
x_{-1}(x)= \begin{cases}\frac{1}{3} x-\frac{1}{3}, & 1 \leqslant x \leqslant 4, \\
-\frac{1}{3} x+\frac{7}{3}, & 4 \leqslant x \leqslant 7,\end{cases} \\
x_{-2}(x)= \begin{cases}\frac{1}{2} x-\frac{3}{2}, & 3 \leqslant x \leqslant 5, \\
-\frac{1}{4} x+\frac{9}{4}, & 5 \leqslant x \leqslant 9,\end{cases} \\
x_{-3}(x)= \begin{cases}x-2, & 2 \leqslant x \leqslant 3, \\
-\frac{1}{5} x+\frac{8}{5}, & 3 \leqslant x \leqslant 8,\end{cases} \\
x_{-4}(x)= \begin{cases}\frac{1}{5} x-\frac{6}{5}, & 6 \leqslant x \leqslant 11, \\
-\frac{1}{2} x+\frac{13}{2}, & 11 \leqslant x \leqslant 13 .\end{cases}
\end{gathered}
$$

In view of (4.2), we get

$$
\begin{gathered}
{\left[x_{0}\right]_{\alpha}=[5+5 \alpha, 14-4 \alpha], \quad\left[x_{-1}\right]_{\alpha}=[1+3 \alpha, 7-3 \alpha], \quad\left[x_{-2}\right]_{\alpha}=[3+2 \alpha, 9-4 \alpha],} \\
{\left[x_{-3}\right]_{\alpha}=[2+\alpha, 8-5 \alpha], \quad\left[x_{-4}\right]_{\alpha}=[6+5 \alpha, 13-2 \alpha] .}
\end{gathered}
$$


From (4.1), it results in a coupled system of difference equation with parameter $\alpha$,

$$
\begin{aligned}
& L_{n+1, \alpha}=\frac{0.3 L_{n-1, \alpha} L_{n-2, \alpha}}{12+3 R_{n-3, \alpha}+6 R_{n-4, \alpha}}, \\
& R_{n+1, \alpha}=\frac{0.3 R_{n-1, \alpha} R_{n-2, \alpha}}{12+3 L_{n-3, \alpha}+6 L_{n-4, \alpha}}, \quad \alpha \in(0,1], n=0,1, \cdots .
\end{aligned}
$$

It is easy to prove that $\left[x_{i}\right]_{\alpha} \subset(0, D / 2 A), i=-4,-3, \cdots, 0$, for $\alpha \in(0,1]$, namely, the conditions of Theorem 3.11 are satisfied. So from Theorem 3.11, we have that the trivial solution $x=\hat{0}$ of (1.1) is asymptotically stable with respect to $\mathrm{D}$ as $\mathrm{n} \rightarrow \infty$ (see Figure 1-4).

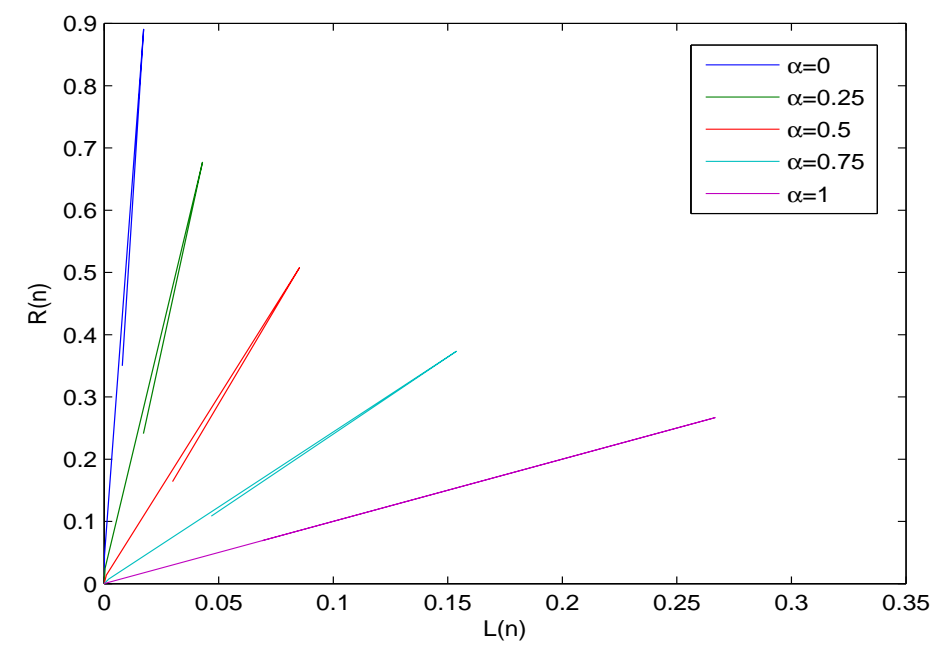

Figure 1: The dynamics of system (4.3).

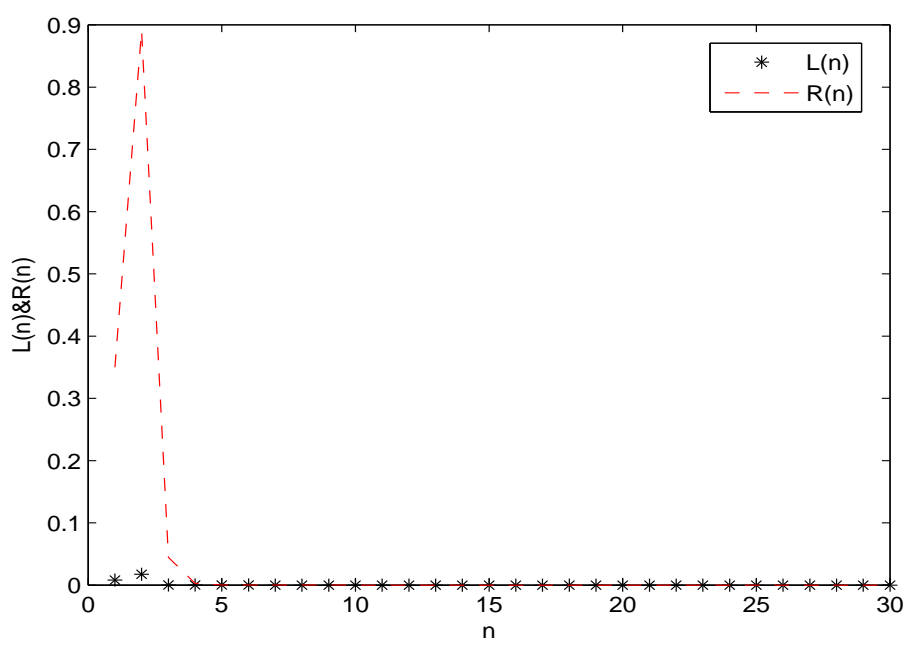

Figure 2: The solution of system (4.3) when $\alpha=0$. 


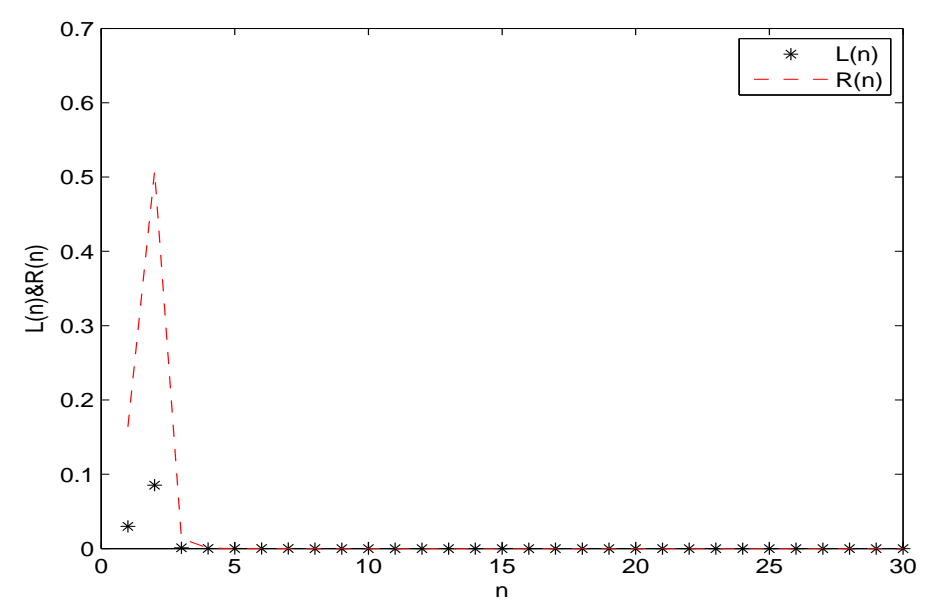

Figure 3: The solution of system (4.3) when $\alpha=0.5$.

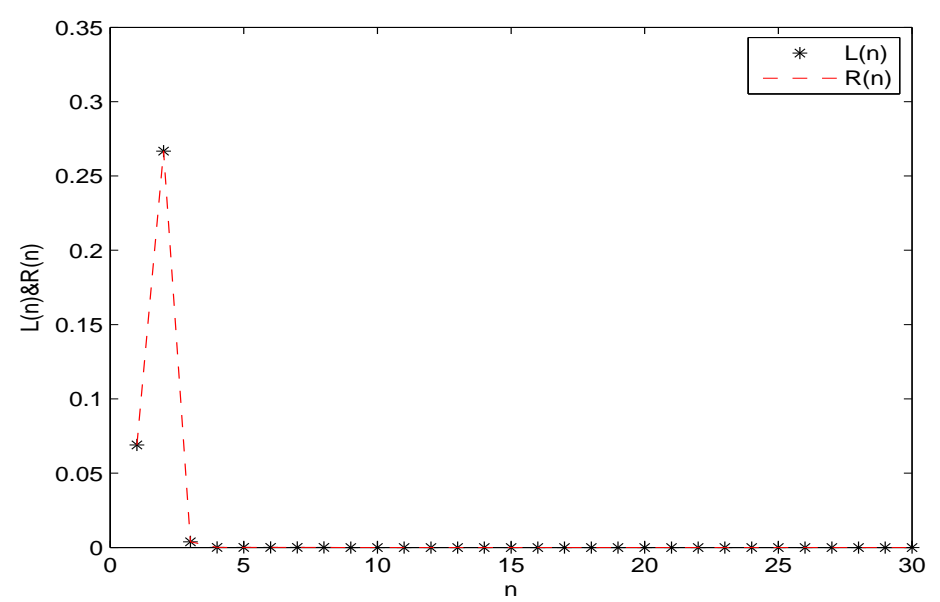

Figure 4: The solution of system (4.3) when $\alpha=1$.

\section{Conclusion}

This paper presents the use of a variational iteration method for systems of nonlinear fuzzy difference equations. This technique is a powerful tool for solving various fuzzy difference equations and can also be applied to other nonlinear differential equations or difference equation in mathematical physics. The numerical simulations show that this method is an effective and convenient one. The variational iteration method provides an efficient method to handle the nonlinear structure. Computations are performed using the software package MATLAB 2014 (a).

In this paper, we have dealt with the dynamics behavior for a class of nonlinear high order fuzzy difference equations. Firstly, the existence and uniqueness of positive fuzzy solutions is proved. Secondly, we also obtain that the nonzero equilibrium points of the corresponding ordinary difference equations (3.12) is unstable by using linearization method. Finally, we find that the trivial solution $\hat{0}$ of (1.1) is stable when the parameters $A, B, C, D$ are positive trivial fuzzy numbers. In particular, some illustrative examples are given to show the effectiveness of the obtained results. In addition, the sufficient conditions that we obtained are very simple, which provide flexibility for the application and analysis of nonlinear fuzzy difference equation. 


\section{Acknowledgment}

This work is supported by Science Fund for Distinguished Young Scholars (cstc2014jc yjjq40004) of China, the National Nature Science Fund (Project nos.11372366 and 61503053) of China, the Natural Science Foundation Project of CQ CSTC (Grant nos. cstc2015jcyj BX0135 and cstc2015jjA20016) of China, and the Postdoctoral Science Foundation (Grant no. 2016m602663) of China.

\section{References}

[1] T. Allahviranloo, S. Salahshour, M. Khezerloo, Maximal- and minimal symmetric solutions of fully fuzzy linear systems, J. Comput. Appl. Math., 235 (2011), 4652-4662. 1

[2] B. Bede, Mathematics of fuzzy sets and fuzzy logic, Studies in Fuzziness and Soft Computing, Springer, Heidelberg, (2013). 2, 3.2

[3] E. Camouzis, G. Ladas, Dynamics of third-order rational difference equations with open problems and conjectures, Advances in Discrete Mathematics and Applications, Chapman \& Hall/CRC, Boca Raton, FL, (2008). 2

[4] K. A. Chrysafis, B. K. Papadopoulos, G. Papaschinopoulos, On the fuzzy difference equations of finance, Fuzzy Sets and Systems, 159 (2008), 3259-3270. 1

[5] E. Y. Deeba, A. De Korvin, Analysis by fuzzy difference equations of a model of $\mathrm{CO}_{2}$ level in the blood, Appl. Math. Lett., 12 (1999), 33-40. 1

[6] E. Y. Deeba, A. De Korvin, E. L. Koh, A fuzzy difference equation with an application, J. Differ. Equations Appl., 2 (1996), 365-374. 1

[7] E. Y. Deeba, A. De Korvin, E. L. Koh, On a fuzzy logistic difference equation, Differential Equations Dynam. Systems, 4 (1996), 149-156. 1

[8] P. Diamond, P. Kloeden, Metric spaces of fuzzy sets, Theory and applications, World Scientific Publishing Co., Inc., River Edge, NJ, (1994). 2

[9] E. M. Elsayed, Solutions of rational difference systems of order two, Math. Comput. Modelling, 55 (2012), 378-384. 1

[10] E. M. Elsayed, On the solutions and periodic nature of some systems of difference equations, Int. J. Biomath., 7 (2014), 26 pages. 1

[11] A. Ghomashi, S. Salahshour, A. Hakimzadeh, Approximating solutions of fully fuzzy linear systems: a financial case study, J. Intell. Fuzzy Systems, 26 (2014), 367-378. 1

[12] E. Hatir, T. Mansour, İ. Yalçinkaya, On a fuzzy difference equation, Util. Math., 93 (2014), 135-151. 1

[13] A. Khastan, New solutions for first order linear fuzzy difference equations, J. Comput. Appl. Math., 312 (2017), 156-166. 1

[14] V. L. Kocić, G. Ladas, Global behavior of nonlinear difference equations of higher order with applications, Mathematics and its Applications, Kluwer Academic Publishers Group, Dordrecht, (1993). 2

[15] V. Lakshmikantham, R. N. Mohapatra, Theory of fuzzy differential equations and inclusions, Series in Mathematical Analysis and Applications, Taylor \& Francis, Ltd., London, (2003). 1

[16] V. Lakshmikantham, A. S. Vatsala, Basic theory of fuzzy difference equations, Special issue in honour of Professor Allan Peterson on the occasion of his 60th birthday, Part I, J. Difference Equ. Appl., 8 (2002), 957-968. 3.10

[17] J. N. Mordeson, P. S. Nair, Fuzzy mathematics, An introduction for engineers and scientists, Second edition, With a foreword by Azriel Rosenfeld, Studies in Fuzziness and Soft Computing, Physica-Verlag, Heidelberg, (2001).

[18] M. Pakdaman, S. Effati, On fuzzy linear projection equation and applications, Fuzzy. Optim. Decis. Mak., 15 (2016), 219-236. 1

[19] G. Papaschinopoulos, B. K. Papadopoulos, On the fuzzy difference equation $x_{n+1}=A+x_{n} / x_{n-m}$, Fuzzy Sets and Systems, 129 (2002), 73-81. 3.1

[20] G. Papaschinopoulos, G. Stefanidou, Boundedness and asymptotic behavior of the solutions of a fuzzy difference equation, Theme: Topology, Fuzzy Sets and Systems, 140 (2003), 523-539. 1

[21] E. C. Pielou, Population and community ecology: principles and methods, CRC Press, London, (1974). 1

[22] E. P. Popov, Automatic regulation and control, (Russian) Nauka, Moscow, (1966). 1

[23] H. Sedaghat, Nonlinear difference equations, Theory with applications to social science models, Mathematical Modelling: Theory and Applications, Kluwer Academic Publishers, Dordrecht, (2003). 2

[24] G. Stefanidou, G. Papaschinopoulos, The periodic nature of the positive solutions of a nonlinear fuzzy max-difference equation, Inform. Sci., 176 (2006), 3694-3710. 1

[25] S. Stević, Behavior of the positive solutions of the generalized Beddington-Holt equation, PanAmer. Math. J., 10 (2000), 77-85. 1

[26] S. Stević, Global stability and asymptotics of some classes of rational difference equations, J. Math. Anal. Appl., 316 (2006), 60-68. 1

[27] C.-Y. Wang, X.-J. Fang, R. Li, On the solution for a system of two rational difference equations, J. Comput. Anal. Appl., 20 (2016), 175-186.

[28] C.-Y. Wang, X.-J. Fang, R. Li, On the dynamics of a certain four-order fractional difference equations, J. Comput. Anal. Appl., 22 (2017), 968-976. 
[29] C.-Y. Wang, S. Wang, W. Wang, Global asymptotic stability of equilibrium point for a family of rational difference equations, Appl. Math. Lett., 24 (2011), 714-718. 1

[30] C.-Y. Wang, S. Wang, Z.-W. Wang, F. Gong, R.-F. Wang, Asymptotic stability for a class of nonlinear difference equations, Discrete Dyn. Nat. Soc., 2010 (2010), 10 pages. 1

[31] C.-X. Wu, B.-K. Zhang, Embedding problem of noncompact fuzzy number space $E^{\sim}$, Fuzzy Sets and Systems, 105 (1999), 165-169. 3.2

[32] Q.-H. Zhang, J.-Z. Liu, Z.-G. Luo, Dynamical behavior of a third-order rational fuzzy difference equation, Adv. Difference Equ., 2015 (2015), 18 pages. 1

[33] Q.-H. Zhang, L.-H. Yang, D.-X. Liao, Behavior of solutions to a fuzzy nonlinear difference equation, Iran. J. Fuzzy Syst., 9 (2012), 1-12. 1

[34] Q.-H. Zhang, L.-H. Yang, D.-X. Liao, On first order fuzzy Ricatti difference equation, Inform. Sci., 270 (2014), $226-236$. 1 